\title{
Effect of the lattice misfit on the equilibrium shape of strained islands in Volmer-Weber growth
}

\author{
José Emilio Prietd* \\ Centro de Microanálisis de Materiales (CMAM), \\ Dpto. Física de la Materia Condensada and Instituto "Nicolás Cabrera", \\ Universidad Autónoma de Madrid, E-28049 Madrid, Spain \\ Ivan Markov \\ Institute of Physical Chemistry, Bulgarian Academy of Sciences, 1113 Sofia, Bulgaria
}

(Dated: November 28, 2018)

\begin{abstract}
We have studied the effect of the misfit on the equilibrium shape of three-dimensional pyramidal islands grown on a foreign substrate in the case of incomplete wetting (Volmer-Weber mode of growth). By means of atomistic simulations using anharmonic interaction potentials, we find that tensile islands have smaller aspect ratios compared with compressed islands owing to their better adhesion to the substrate. The average strains of consecutive layers decrease faster with thickness in compressed than in tensile islands. The strains decrease rapidly with thickness, with the consequence that above a certain height, the upper layers of the pyramid become practically unstrained and do not contribute to a further reduction in the top base. As a result, the truncated pyramids are not expected to transform into full pyramids. Our results are in good agreement with experimental observations in different systems.
\end{abstract}

PACS numbers: 68.35.Md, 68.43.Hn, 68.55.A-, 68.35.Np

Elastic strains play an important role in the growth of heteroepitaxial thin films. In the case of complete wetting of the substrate by the film material, the elastic strain due to the lattice misfit results in a transition from planar to three-dimensional (3D) island growth beyond a critical thickness $\frac{1,2}{2}$ This is the well known StranskiKrastanov (SK) mode of growth, which is widely used to produce quantum dots in semiconductor systems such as $\mathrm{Ge} / \mathrm{Si}^{\underline{3}}$ and InAs $/ \mathrm{GaAs}^{4}$. In the case of incomplete wetting (non-zero wetting angle), 3D islanding takes place from the very beginning of the deposition, irrespective of the value of the misfit. Nevertheless, in this case of Volmer-Weber (VW) growth, the misfit can play a crucial role in determining the equilibrium shape and, in particular, the aspect ratio of the 3D islands. The two cases mentioned above differ considerably. In the SK mode, complete wetting and misfit strain operate in different directions: complete wetting favours planar growth, while strain favours clustering. This gives rise to an instability of planar growth against 3D islanding. In the VW mode both the incomplete wetting and the effect of misfit strain favor islanding.

For the reasons given above, the equilibrium shape of islands on misfitting substrates has been the object of intensive studies ${ }^{-111}$. The dependence of the equilibrium shape in both SK and VW modes has been considered in detail by Müller and Kern.12-14 In the case of VW growth, they found that box-shaped crystals thicken with increasing value of the misfit. A qualitatively similar conclusion was reached in Ref. 15] under the condition that the crystal does not relax and is homogeneously strained to fit the substrate. In the case of pyramidal islands, the above authors found that the upper base shrinks and the island shape can change from truncated to complete pyramids with increasing misfit. It has been found in addition that the equilibrium shape depends on the square of the lattice misfit thus being independent of the misfit sign. ${ }^{14}$ Müller and Kern explained this result by neglecting surface stress effects in their calculations.

In the present paper we study the effect of the lattice misfit, and in particular, of its sign on the equilibrium shape of pyramidal crystals. We performed atomistic simulations considering square-based pyramidal islands with fcc atomic structure and (100) orientation, located on a substrate to which periodic boundary conditions are applied. The equilibrium shape of fcc crystals considering only nearest neighbor interactions is a truncated octahedron or a cube-octahedron enclosed by six (001) and eight (111) faces with equal edge lenghts. $\underline{16}$ The wetting function (or adhesion parameter) is defined as 17,18

$$
\phi=1-\frac{\psi^{\prime}}{\psi}
$$

where $\psi^{\prime}$ and $\psi$ are the substrate-deposit (adhesion) and deposit-deposit (cohesion) atomic interaction energies, respectively. If the wetting function is smaller than 0.25 at zero misfit, the lateral cubic faces do not appear and the equilibrium shape remains a simple truncated pyramid (Fig. 1). The lattice misfit decreases the degree of wetting and contributes to an effective increase in the wetting function, $\frac{19,20}{2}$ For this reason, we restrict ourselves to values of the wetting in the interval $\phi=0 \div 0.2$.

We have performed simple minimization calculations. The atoms in the islands interact through a pair potential whose anharmonicity can be varied by adjusting two constants $\mu$ and $\nu(\mu>\nu), 21,22$

$$
V(r)=V_{0}\left[\frac{\nu}{\mu-\nu} e^{-\mu(r-b)}-\frac{\mu}{\mu-\nu} e^{-\nu(r-b)}\right]
$$


where $b$ is the equilibrium atom separation. For $\mu=2 \nu$ the potential adopts the familiar Morse form. It is worth to note that these kinds of potentials, due to their spherical symmetry, are better suited to describe the bonding in metals. By contrary, semiconductors are characterized by directional, covalent bonds and bonding angles need to be taken into account. However, the potentials appropriate for semiconductors are also anharmonic ${ }^{23}$, so we expect our results using anharmonic potentials to be qualitatively valid for those materials as well.

Energy minimization is performed by allowing the atoms to relax until the forces fall below some negligible cutoff value. In spite of its simplicity, the above potential includes the necessary features to describe real materials (theoretical strength and anharmonicity) by varying the constants $\mu, \nu$ and $V_{0}$. We consider interaction only in the first coordination sphere and simulate both rigid and relaxable substrates, in which a given number of layers $S$ are allowed to relax. The initial positions of the atoms correspond to the centers of the potential troughs provided by their neighbors underneath; those neighbors are not updated when atomic displacements approach values close to one half of the interatomic distance, so our model is not appropriate for the description of configurations containing misfit dislocations. The interatomic spacing in the substrate material is $a$ so that the lattice misfit is given by $f=(b-a) / a$. The case of positive misfits corresponds to the lattice parameter of the deposit being larger than that of the substrate, so the material in the islands is compressed. The atoms interact with their lateral neighbors mainly through the stronger repulsive branch of the potential. In the opposite case, the islands are tensile and the atoms interact with their lateral neighbors with the weaker attractive branch of the potential. As a result, the surface stress of the side walls should differ in compressed and tensile crystallites, or in other words, they should relax in different degrees. The side walls of compressed islands are expected to relax by a greater amount compared with tensile islands.

In addition, the misfit sign affects the adhesion of the crystals to the substrate in a different way. In compressed islands the atoms of the first atomic layer are strongly displaced from the bottom of the corresponding potential troughs of the substrate owing to the stronger repulsion of the lateral neighbors. As a result, the adhesion is reduced. On the contrary, the first-layer atoms in tensile islands are only slightly displaced from the bottoms of the respective potential troughs of the substrate and the adhesion is stronger compared with compressed islands at the same absolute value of the lattice misfit 26,27 .

In order to determine the equilibrium shape of the islands, we apply the following procedure. We consider islands of different heights and bases with shapes close to squares, either of $n \times n$ or of $n \times(n+1)$ atoms, where $n$ is an integer. Allowing the structures to relax, we calculate the total energy per atom of the islands, for heights $h$ varying by 1 monolayer (ML), as a function of the total number of atoms $N$ in the pyramid. Due to the dis-

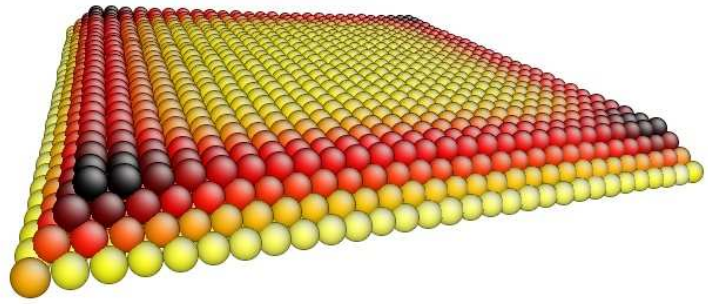

FIG. 1: (Color online) Epitaxial fcc(100) island of $-7 \%$ misfit on a rigid substrate. It has the shape of a truncated square pyramid with $30 \times 30$ atoms in the base plane and a height of 4 monolayers. The color scale denotes the level of hydrostatic strain as given by the atomic local strain tensor ${ }^{24}$ and has been represented using the AtomEye software ${ }^{25}$. Strain is highest at the center of the island and at the bottom layer and it is relaxed at island edges and at the topmost layer.

crete character of the island sizes produced in this way, we need to interpolate between the two closest values at the corresponding height to the desired total number of atoms, e.g. $N=1000$. In this way, we find the height of the island with lowest energy. For example, Fig. 2 shows that four-layers thick islands have lowest energy when the number of atoms is smaller than 300. At $N>300$ the lowest energy thickness is 5 MLs. We then calculate the lowest energy heights of islands at a given constant number of atoms and for different values of the lattice misfit. Once we know the lowest energy heights we determine the edge lengths of the lower, $n$, and upper, $n^{\prime}$, bases from the relation $n^{\prime}=n-h+1$ and from the formula for the total number of atoms $N(n, h)=\sum_{k=1}^{n} k^{2}-\sum_{k=1}^{n-h} k^{2}$ as a function of $n$ and $h$. The height $h$ is measured in number of monolayers and $n$ and $n^{\prime}$ give the number of atoms in the upper and lower edges, respectively. Thus, the full pyramid is characterized by $n^{\prime}=1$ and $h / n=1$.

The simplest expression for the equilibrium shape of the pyramid is $h / n^{\prime}=4 \phi$, where $\phi$ is the wetting function defined above and the coefficient 4 reflects the coordination of an atom on the (001) surface. This formula has been derived by comparing the works per atom to evaporate whole atomic planes of the lateral pyramidal faces and the upper base, a method which had been introduced by Stranski and Kaischew ${ }^{28}$ (an excellent recent review is given in Ref. 29). However, we will illustrate our results in terms of plots of the ratio $h / n$ vs. the lattice misfit because the ratio $h / n^{\prime}$ tends to the island height $h$, which depends on the number $N$ of atoms in the pyramid when it becomes complete and the upper base disappears, while the ratio $h / n$ tends to unity.

Figure 3 demonstrates our main result. It shows the dependence of the aspect ratio $h / n$ of islands of minimum energy on the value of the misfit, both negative and positive ones. The islands consist of small (a) $N=250$ and large (b) $N=1000$ number of atoms. The substrate 


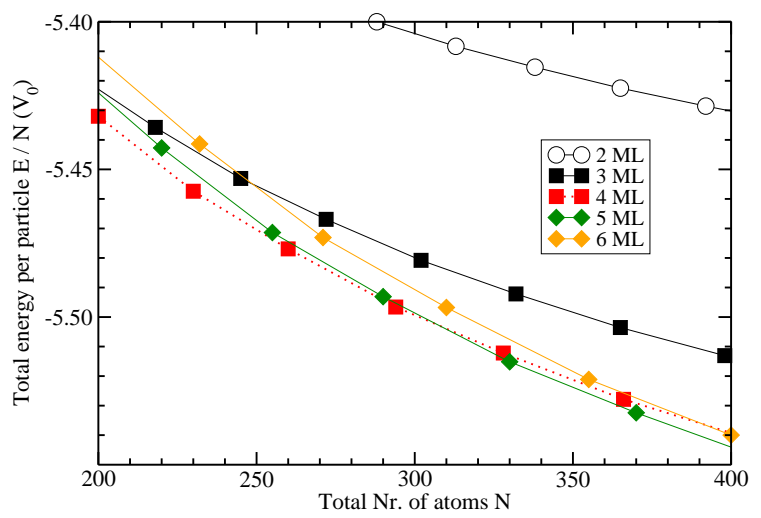

FIG. 2: (Color online) Dependence of the total energy per atom as a function of the number of atoms $N$ in the pyramid for islands with different heights varying through one monolayer. As seen islands with $N<300$ have lowest energy when they are four monolayers high whereas the lowest energy height is 5 monolayers at $N>300$. The wetting parameter is $\phi=0.2$, the lattice misfit $f$ is zero and a rigid substrate was assumed in the simulations.

is assumed rigid. Several important conclusions can be reached. First, the lattice misfit has a great effect on the equilibrium shape. The aspect ratio increases steeply beyond a moderate misfit of about $3 \%$. The smaller the wetting function (i.e. the stronger the adhesion), the stronger the effect of the misfit. Or, the stronger the tendency to $3 \mathrm{D}$ islanding due to the weaker wetting, the smaller is the effect of the lattice misfit. The effect of the misfit is greatest at $\phi \rightarrow 0$. As mentioned above, the reason is that both effects are complementary.

Second, it is seen that a positive misfit has a perceptibly greater effect on the equilibrium shape than a negative one of the same absolute value. This is due to the stronger repulsive forces of the interatomic potential and to the weaker adhesion. In tensile overgrowth the increase in the aspect ratio is delayed to greater absolute values of the misfit. And more important, increasing the lattice misfit does not lead to disappearance of the upper base, $h / n \rightarrow 1$, as predicted by Müller and Kern ${ }^{14}$. In both cases of positive and negative misfit the aspect ratio $h / n$ goes to a saturation value of about 0.75 irrespective of the island size and misfit sign.

The curves $h / n$ vs misfit show an initial increase and an inflection point. Beyond this point the curves tend to a saturation value lower than unity as mentioned above. Note that at large absolute values of the misfit the interface between the crystallites and the substrate is expected to be resolved in a cross grid of misfit dislocations. The arrows in Fig. 3 show the approximate values of the misfit at which dislocations are introduced in compressed islands. The tensile islands, on the other hand remain coherent. Although our model, as explained above, is not well suited for the description of dislocated configurations, it seems clear that the tendency to saturation begins long before the introduction of dislocations. Fur-
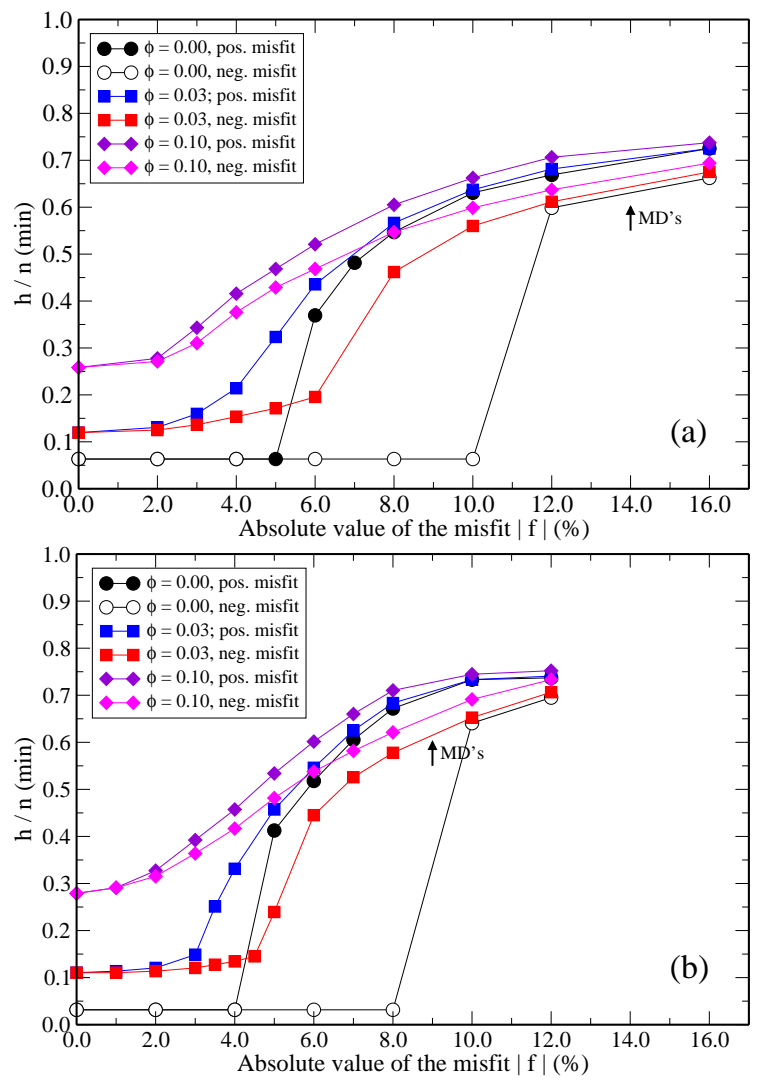

FIG. 3: (Color online) Misfit dependence of the aspect ratio $h / n$ for different values of the wetting function $\phi=0.0,0.03$ and 0.10 and a total number of atoms in the pyramid of (a) $N=250$, (b) $N=1000$. A rigid substrate was assumed in the calculations. The arrows mark the appearance of misfit dislocations in the growing islands.

thermore, since both 3D clustering as well as the introduction of misfit dislocations are (possible competing) mechanisms that contribute to the relaxation of epitaxial strain, we can conclude that the introduction of misfit dislocations is not the basic reason for the islands to remain truncated pyramids at the sizes studied so far. As will be shown below the main reason is the fast decrease in the strain with the pyramid height.

We can understand the different behavior of the aspect ratio at positive and negative misfits as follows. As shown in $1+1$ dimensional models $\frac{1930}{}$, the thermodynamic driving force for 3D islanding in coherent StranskiKrastanov growth is the reduced adhesion of the initial $2 \mathrm{D}$ islands to the wetting layer. This is due to the displacements of the atoms located closer to the islands edges from the respective potential troughs of the substrate (the wetting layer). Thus the average adhesion parameter is non-zero and 3D islanding is thermodynamically favored. A more detailed study of the adhesion parameter as a function of the misfit sign has shown that compressed islands display larger values of the adhesion parameter (weaker wetting) compared with tensile islands. Hence, greater absolute values of the (negative) 
misfit in tensile islands are required in order to reach the same values of the adhesion parameter as compared to compressed islands.

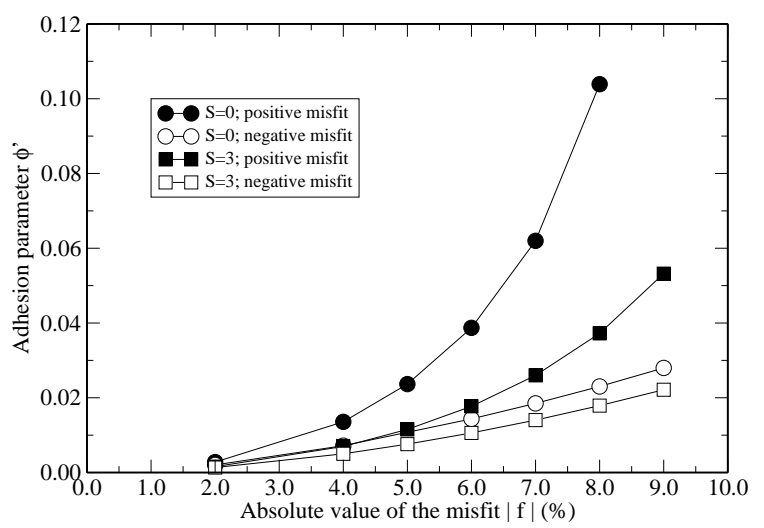

FIG. 4: Misfit dependence of the adhesion parameter, $\phi^{\prime}(f)$, for compressed and tensile islands for the case of $\phi=0.0$ (SK mode of growth). Results are shown for different numbers $S$ of substrate layers allowed to relax: circles for $\mathrm{S}=0$ (i.e., rigid substrate) and squares for $\mathrm{S}=3 \mathrm{ML}$. The islands are 5 ML high and contain a total amount of $N=990$ atoms.

The same result is obtained in our $2+1$ dimensional model as demonstrated in Fig. 4 for the StranskiKrastanov growth. Here the wetting parameter $\phi$ due to different bonding, as defined by Eq.(1), equals zero and we can define a mean wetting parameter $\phi^{\prime}$ as

$$
\phi^{\prime}=1-\frac{U_{\mathrm{adh}}}{\left(-4 V_{0}\right)},
$$

where $U_{\text {adh }}$ is the mean interaction energy between an atom at the bottom layer of the island and the substrate, which equals $-4 V_{0}$ at zero misfit. The tensile islands always display better adhesion to the substrate. Although the difference in adhesion is greater in rigid substrates compared with relaxed ones, the effect of the potential anharmonicity remains significant. The adhesion parameter for tensile islands remains close to zero (complete wetting).

In the case of Volmer-Weber growth the effective adhesion parameter in misfitting overgrowth, $\phi_{\text {eff }}$, is thus composed of two parts. The first part is due to the difference in bonding and is given by Eq. (11), while the second part is due to the lattice misfit as discussed above. We can thus write

$$
\phi_{\mathrm{eff}}(f)=\phi+\phi^{\prime}(f)
$$

where the second term in the right-hand side depends solely on the lattice misfit, $f$, so that $\phi^{\prime}(0)=0$. The inequality $\phi^{\prime}(+|f|)>\phi^{\prime}(-|f|)$ and in turn $\phi_{\text {eff }}(+|f|)>$ $\phi_{\text {eff }}(-|f|)$ explains the results shown in Fig. 3 .

We now study the strain distribution in tensile and compressed islands on a substrate which is allowed to relax. Fig. 5(a) shows the distribution of the strains in a compressed crystallite $(f=+4 \%)$. The crystal is $5 \mathrm{MLs}$ thick and the same number of monolayers of the substrate are allowed to relax. As seen, the 4 th and the 5th MLs of the substrate are very weakly strained. The strains in the substrate below the island do not exceed $1 \%$ whereas those in the first layer of the crystallite are close to $-3 \%$.
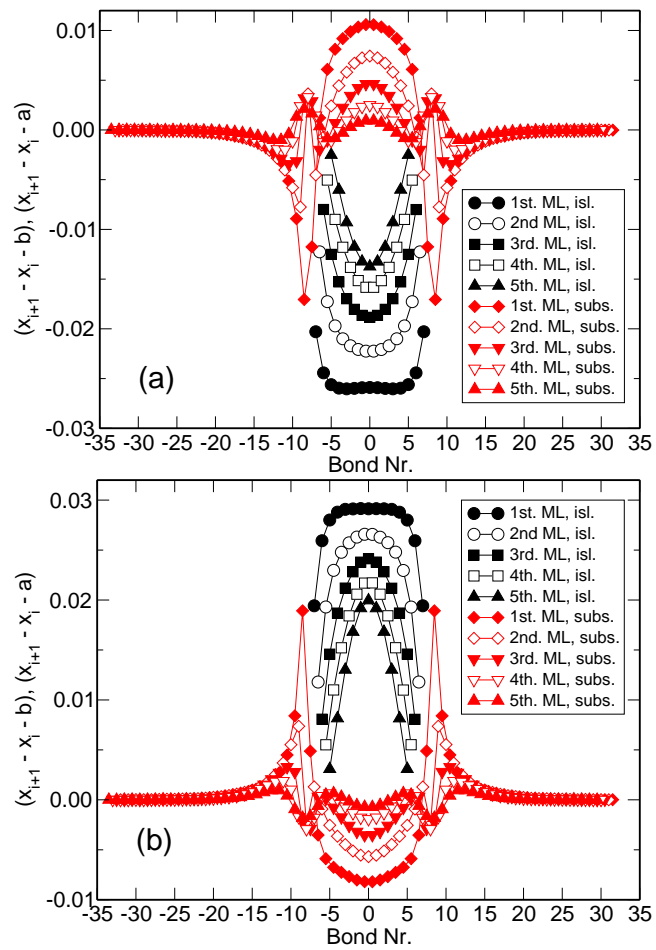

FIG. 5: (Color online) Distribution of the lateral bond strains in the center of the crystal and in the layers of the substrate underneath for misfits of (a) $f=4 \%$ and (b) $f=-4 \%$. Strains are referred to the lattice parameters of crystal $a$ and substrate $b$, respectively. The wetting parameter due to the different chemical nature of substrate and adsorbate is $\phi=0.03$, islands of $5 \mathrm{ML}$ height and 990 atoms were considered and $5 \mathrm{ML}$ of the substrate were allowed to relax.

Figure 5(b) shows the corresponding strain distribution in tensile island at the same absolute value of the misfit $(f=-4 \%)$. The distribution is qualitatively the same. The strains in the island are again much larger than those in the substrate. This is easy to understand having in mind that the substrate crystal has no free edges and the substrate atoms are highly constrained by their lateral neighbors. As a result, the total strain energy stored in both compressed and tensile islands is about two orders of magnitude larger than the strain energy stored in the substrate. The important conclusion we can extract is that the substrate behaves as relatively "stiff" against the epitaxial strain induced by the overgrowth and can be safely considered rigid in comparison with the deformations of the islands. This justifies the results in Fig. 3 where the substrate was assumed to be rigid.

A more careful inspection of Fig. 5 shows that the lateral strains of the separate layers in compressed islands 
are always smaller than the corresponding strains in tensile islands. In addition, the strains of the separate layers decrease with layer height, this decrease being larger in compressed than in tensile islands (Fig. 66). In other words, compressed islands tend faster to achieve the bulk lattice parameter of the overgrowth material compared with tensile islands.

However, the height distribution of the strain energies of the separate layers shows an unexpected behavior (Fig. 7). The strain energies of the layers beyond the second one in tensile islands become larger than those in compressed layers. This can be understood as follows. The more strained the layer the more significant is the effect of the potential anharmonicity. The first layers are highly strained and the steeper repulsive branch of the potential leads to a very high strain energy of the compressed island. The tendency of atoms to relax the strain is thus higher in compressed overlayers and this leads to smaller values of the strain in spite of the higher amount of strain energy as compared to tensile islands at the same absolute value of the lattice misfit. Upper layers become less and less strained and the anharmonicity cannot overcompensate the smaller absolute values of the strains. As a result, the more strained upper layers in tensile islands possess also a larger amount of strain energy.

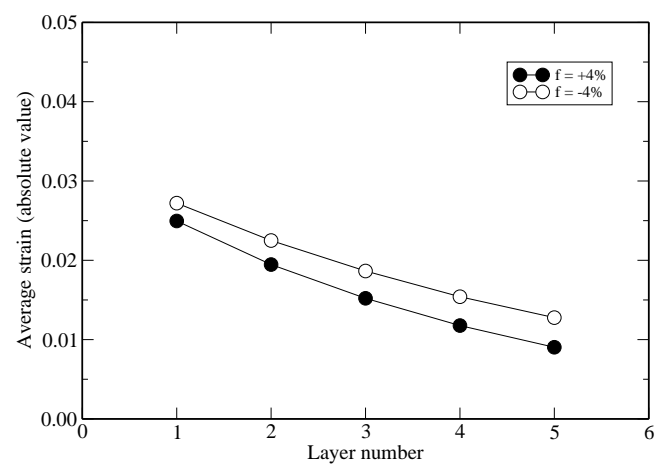

FIG. 6: Average strains of consecutive layers in compressed and tensile pyramids with $+4.0 \%$ and $-4.0 \%$ lattice misfit, respectively. Islands are $5 \mathrm{ML}$ high and contain 990 atoms, the wetting parameter is $\phi=0.03$ and $5 \mathrm{ML}$ of the substrate were allowed to relax.

Figure 5 also shows that the first layer is practically pseudomorphic with the substrate (the strains are nearly equal to the misfit taken with opposite sign) with the exception of a few edge bonds. On the contrary, most of the bonds in the uppermost (fifth) layer are much less strained than the nominal misfit showing that the strains rapidly goes to zero. It follows that the upper layers of a complete pyramid are practically fully relaxed and so they do not contribute to the accumulation of strain in the crystallite. For this reason, the crystallites do not transform to full pyramids for reasonable values of the misfit as expected from the continuous and harmonic theory of Müller and Kern. The presence of misfit dislocations favors further relaxation of the upper layes, but they are expected to play a secondary role in preventing the transformation of the small islands into full pyramids. An additional argument is that misfit dislocations are introduced in tensile islands always at misfit values where saturation takes place. In order to further clarify the role of the anharmonicity of the potential, we also studied the island's aspect ratio as a function of the lattice misfit using harmonic potentials of the same spring constants as the anharmonic ones previously discussed. The curves (not shown) obtained for positive and negative misfits nearly overlap and are located as expected between the curves corresponding to the anharmonic potentials, slightly closer to the curve of positive misfit.

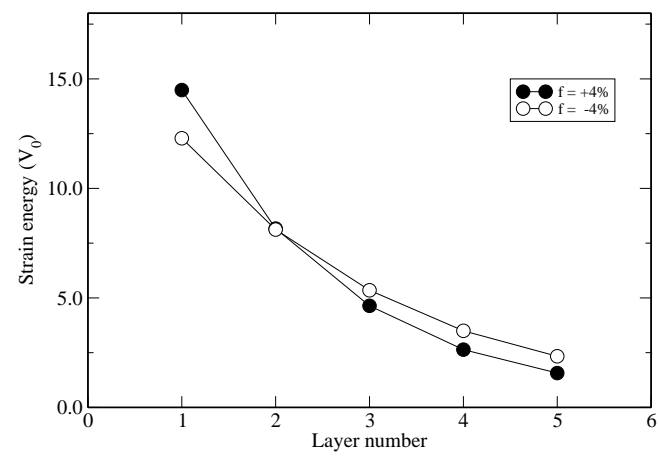

FIG. 7: Strain energy of consecutive layers in compressed and tensile pyramids with $+4.0 \%$ and $-4.0 \%$ lattice misfit, respectively. Islands are $5 \mathrm{ML}$ high and contain 990 atoms, the wetting parameter is $\phi=0.03$ and $3 \mathrm{ML}$ of the substrate were allowed to relax.

It is worth to note that the equilibrium aspect ratio of the $3 \mathrm{D}$ islands in the case of complete wetting (SK mode) follows the same trend as in the case of VW growth mode (Fig. 3). The only difference is that the initial islands are single-layer islands $(\phi=0)$ which then transform into multilayer islands 31 . The only physical reason for incomplete wetting is the lattice misfit which leads to displacements of the overlayer atoms from the bottom of the substrate potential troughs $\frac{19,20}{}$ and thus to a weakening of the interfacial bonding.

Experimental observations of equilibrium shape of islands on misfitting substrates, in the appropriate ranges of parameters to compare with the results of our model, are scarce in the literature. We will not discuss here the results concerning the equilibrium shape of metal clusters on $\mathrm{MgO}$ (for a review see Ref. 16). One of the reasons is that wetting functions are greater than 0.5 and so the equilibrium shapes are not simple pyramids. Another reason is that all metals studied $(\mathrm{Ni}, \mathrm{Pd}, \mathrm{Pt})$ show a negative misfit with the substrate 32 . Nevertheless, smallest particles of $\mathrm{Pd}$ on $\mathrm{MgO}$ ( $\mathrm{f}=-6 \%$ ) studied showed an equilibrium shape of a truncated pyramid, $h / n \approx 0.7 \underline{33}$.

Goldfarb et al.$^{34}$ found that the equilibrium shape of small crystals of $\mathrm{TiSi}_{2}$ on $\mathrm{Si}(111)$ is a flat hexagonal pyramid with the $(01 \overline{3})$ atomic plane parallel to $\mathrm{Si}(111)$. The misfit in the two directions $<112>$ and $<110>$ amounts to $5 \%$ and $10 \%$, respectively. Silly 
and Castell studied a completely different system: Fe on $\mathrm{SrTiO}_{3}(001)^{35}$. After annealing, the equilibrium shape of bcc-Fe islands is a square pyramid with an aspect ratio $l / h=1.2$ irrespective of the island size, where $l$ is the edge length of the upper base. The side walls make an angle of $45^{\circ}$ with the substrate so that the aspect ratio $h / L$ amounts to 0.31 , where $L$ is the edge length of the lower base. Nowicki et al. found that the equilibrium shape of $\mathrm{Pb}$ islands on $\mathrm{Ru}(001)$ corresponds to a truncated pyramid with the (111) face as the upper base $e^{36,37}$. So in all cases discussed above, the equilibrium shape is a truncated pyramid in accordance with the results of the present paper.

In conclusion, using anharmonic interaction potentials, we have found that compressed islands show larger values of the aspect ratio than tensile ones at the same absolute value of the misfit owing to its larger effective adhesion parameter. Atoms belonging to the first layer of compressed islands show larger displacements from the bottom of the potential troughs of the substrate due to the stronger interatomic repulsive forces, which leads to weaker adhesion of the islands to the substrate. On the contrary, the weaker attractive branch of the potential acts between the atoms in tensile islands and the effective wetting parameter is smaller. Due to the same reasons, compressed islands are less strained compared with tensile ones and tend to attend the bulk lattice parameter more rapidly with increasing island thickness. As a result, the upper layers of the pyramids are practically unstrained and they cannot transform to full pyramids with increasing values of the lattice misfit. The strain energy stored in the substrate is about two orders of magnitude smaller than in the islands due to the constraint of the atoms by their lateral neighbors. As a result, for many calculations, the substrate can usually by assumed as rigid.

\section{Acknowledgments}

This work was financed by project FIS2008-01431 of the Spanish MiCInn; J.E. Prieto also acknowledges support by its program "Ramón y Cajal".
* Electronic address: joseemilio.prieto@uam.es

† Electronic address: imarkov@ipc.bas.bg

1 E. Bauer, Z. Kristallographie 110, 372 (1958).

2 R. Peierls, Phys. Rev. B 18, 2013 (1978).

3 D. J. Eaglesham and M. Cerullo, Phys. Rev. Lett. bf 64, 1943 (1990).

${ }^{4}$ B. A. Joyce and D. D. Vvedensky, Mater. Sci. Eng. Reports 46, 127 (2004).

5 M. E. Thompson, C. S. Su, P. W. Voorhees, Acta Metallurgica et Materialia 42, 2107 (1994).

6 B. J. Spencer, J. Tersoff, Phys. Rev. Lett. 79, 4858 (1997).

7 N. Moll, M. Scheffler, E. Pehlke, Phys. Rev. B 58, 4566 (1998).

8 I. Daruka, A.-L. Barabási, Phys. Rev. Lett. 79, 3708 (1997).

9 H. Uemura, M. Uwaha, Y. Saito, J. Phys. Soc. Jpn. 71, 1296 (2002).

${ }^{10}$ Ch. Duport, J. Villain, C. Priester, in: Surface Diffusion: Atomistic and Collective Processes, ed. by M. C. Tringides, NATO ASI Series, Series B: Physics, Vol. 360, (Plenum Press, 1997), p. 191.

11 O. E. Shklyaev, M. J. Miksis, P. W. Voorhees, J. Mech. Phys. Solids 54, 2111 (2006).

12 P. Müller and R. Kern, Appl. Surf. Sci. 102, 6 (1996).

${ }_{13}$ P. Müller and R. Kern, J. Cryst. Growth 193, 257 (1998).

14 P. Müller and R. Kern, Surf. Sci. 457, 229 (2000).

15 I. Markov, Crystal Growth for Beginners, Fundamentals of Nucleation, Crystal Growth and Epitaxy, World Scientific, Singapore, 1995, p. 75.

16 C. R. Henry, Progr. Surf. Sci. 80, 92 (2005).

17 R. Kaischew, Commun. Bulg. Acad. Sci. (Phys.) 1, 100 (1950).

18 R. Kaischew, Fortschr. Miner. 38, 7 (1960).

19 E. Korutcheva, A. M. Turiel, I. Markov, Phys. Rev. B 61, 16890 (2000).
20 J. E. Prieto and I. Markov, Phys. Rev. B 66, 073408 (2002).

21 I. Markov, Phys. Rev. B 48, 14016 (1993).

22 J. E. Prieto and I. Markov, Phys. Rev. Lett. 98, 176101 (2007).

23 J. Tersoff, Phys. Rev. Lett. 61, 2879 (1988); Phys. Rev. B 37, 6991 (1988); Phys. Rev. B 38, 9902 (1988).

24 F. Shimizu, S. Ogata and J. Li, Materials Transactions 48 2923 (2007).

25 J Li, Modelling Simul. Mater. Sci. Eng. 11, 173 (2003).

26 I. Markov and A. Milchev, Surf. Sci. 136, 519 (1984).

27 I. Markov and A. Milchev, Surf. Sci. 145, 313 (1984).

28 I. N. Stranski and R. Kaischew, Z. Phys. Chem. 26, 100, 114, 132 (1934).

29 V. L. Tassev and D. F. Bliss, J. Cryst. Growth 310, 4209 (2008).

30 I. Markov and J. E. Prieto, Atomistic Aspects of Epitaxial Growth, NATO Science Series, II. Mathematics, Physics and Chemistry - Vol. 65, M. Kotrla, N. I. Papanicolaou, D. D. Vvedensky, L. T. Wille eds., (Kluwer Academic, Dordrecht, 2002), p. 411.

31 J.E. Prieto and I. Markov, Phys. Rev. B 72, 205412 (2005).

32 C. Mottet and J. Goniakowski, J. Comput. Theor. Nanosci. 4, 326 (2007).

33 S. Giorgio, C. R. Henri, C. Chapon, J. M. Penisson, J. Cryst. Growth 100, 254 (1990).

34 I. Goldfarb, G. Cohen-Taguri, S. Grossman, M. Levinshtein, Phys. Rev. B 72, 075430 (2005).

35 F. Silly and M. Castell, Appl. Phys. Lett. 87, 063106 (2005).

36 M. Nowicki, C. Bombis, A. Emundts, H. P. Bonzel, P. Winblatt, New J. Phys. 4, 60 (2002).

37 H. P. Bonzel, D. K. Yu, M. Scheffler, Appl. Phys. A 87, 391 (2007). 